\title{
Resonant behavior observed in electron field emission from acid functionalized multiwall carbon nanotubes
}

\author{
S. M. Lyth and S. R. P. Silva ${ }^{\text {a) }}$ \\ Nanoelectronics Centre, Advanced Technology Institute, University of Surrey, Guildford, Surrey GU2 7XH, \\ United Kingdom
}

(Received 25 November 2008; accepted 11 February 2009; published online 24 March 2009)

\begin{abstract}
Acid functionalized multiwall carbon nanotube ink was deposited onto carbon fiber fabric via dip coating. Repeatable staircaselike current-field curves were observed in the field emission data. These atypical curves are attributed to resonant tunneling through localized surface states in a quantum well structure, which arises due to the presence of the surface carboxylic functional group. (C) 2009 American Institute of Physics. [DOI: 10.1063/1.3094755]
\end{abstract}

Field emission (FE) is the emission of electrons from a solid by the application of a large electric field via quantum tunneling through an approximately triangular potential barrier. Interesting quantum phenomena such as Coulomb blockade $^{1}$ and resonant tunneling (RT) have been observed in FE. RT solid-state semiconductor devices have important applications in high frequency oscillators and mixers and in RT diodes. ${ }^{2}$ However, RT in FE has not been widely observed. In 1996, Kryuchenko and Litovchenko ${ }^{3}$ performed a computer simulation of FE from multilayer cathodes, resulting in negative differential resistance caused by RT of electrons through quantum well subbands. The same group later observed experimentally RT in FE from similar multilayered structures. ${ }^{4}$ Similarly, negative differential resistance has been observed in FE from amorphous carbon based multilayer structures. ${ }^{5}$

Carbon nanotubes (CNTs) were first observed in 1952 as an industrial contaminant ${ }^{6}$ and recognized in context in 1991 as molecular microtubules of graphitic carbon. ${ }^{7}$ Their high strength, ${ }^{8}$ great length, ${ }^{9}$ and ability to be spun into fibers could lead to applications in materials science. ${ }^{10}$ They have high electrical and thermal conductivity, making them ideal candidates for use in electrical devices ${ }^{11,12}$ such as sensors. ${ }^{13,14}$ CNTs can be grown over large areas ${ }^{15}$ and at low temperatures,${ }^{16}$ making them perfect for large area applications on temperature-sensitive substrates. Of interest in this work are the FE properties of CNTs. A vast international body of work has been published on their application in FE displays. ${ }^{17-20}$ CNTs can be solubilized by an acid oxidative chemical treatment, resulting in the formation of carboxylic acid groups attached to the CNT ends and sidewalls, ${ }^{21}$ which readily interact with water molecules via hydrogen bonding. Acid treatment also purifies CNTs, dissolving catalytic particles and amorphous carbon. ${ }^{22}$ Solubilization opens the door to a variety of cost effective, scalable, room temperature deposition techniques, such as spin-coating and ink-jet printing; thus CNT inks can be deposited onto a plethora of different substrates. The excellent FE properties of CNT ink on paper substrates have recently been explored. ${ }^{23}$

In previous work by the authors, the surface of multiwall CNTs (MWNTs) was chemically modified, resulting in a change in work function ${ }^{24}$ and thereby a change in the FE properties. ${ }^{25}$ Subsequently, close analysis of the FE charac-

\footnotetext{
${ }^{a)}$ Electronic mail: s.silva@surrey.ac.uk.
}

teristics of acid-oxidized MWNTs (o-MWNTs) revealed unexpected staircaselike increases in the current-field curves. Here, these atypical curves are analyzed, and the results are discussed in terms of RT through localized surface states.

High purity, $10 \mathrm{~nm}$ diameter MWNTs were purchased from Nanocyl. Acid oxidization was performed as previously described. ${ }^{25}$ Carbon fiber fabric (Ballard) was used as a substrate due to its high conductivity and good FE properties, and it was rendered hydrophilic via a $10 \mathrm{~s}$ oxygen plasma microwave treatment. This results in the formation of oxygen containing moieties, ${ }^{26}$ promoting wetting between the o-MWNT ink and the carbon fiber. Hydrogen bonding is also expected to provide strong adhesion between the carbon fiber and the o-MWNTs. After plasma treatment, the carbon fabric was dip-coated in o-MWNT ink and then baked at $100{ }^{\circ} \mathrm{C}$ for $20 \mathrm{~min}$. FE characterization was conducted as previously described. $^{25}$

Figures 1(a)-1(d) show scanning electron microscope (SEM) images of the samples. Figure 1(a) shows the pristine carbon fiber fabric, Fig. 1(b) shows the carbon fabric after oxygen plasma treatment, while Figs. 1(c) and 1(d) show the carbon fabric after dip-coating. After dip-coating, a continuous layer of o-MWNTs is observed over the whole carbon fiber surface. Therefore electron emission can be assumed to

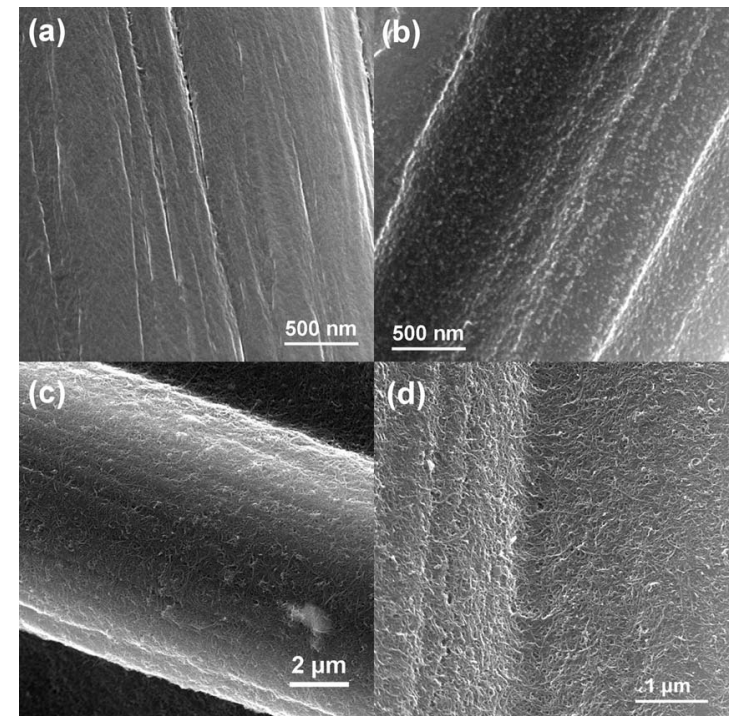

FIG. 1. SEM images of (a) pristine carbon fiber, (b) oxygen plasma treated carbon fiber, and [(c) and (d)] carbon fiber coated with o-MWNTs. 

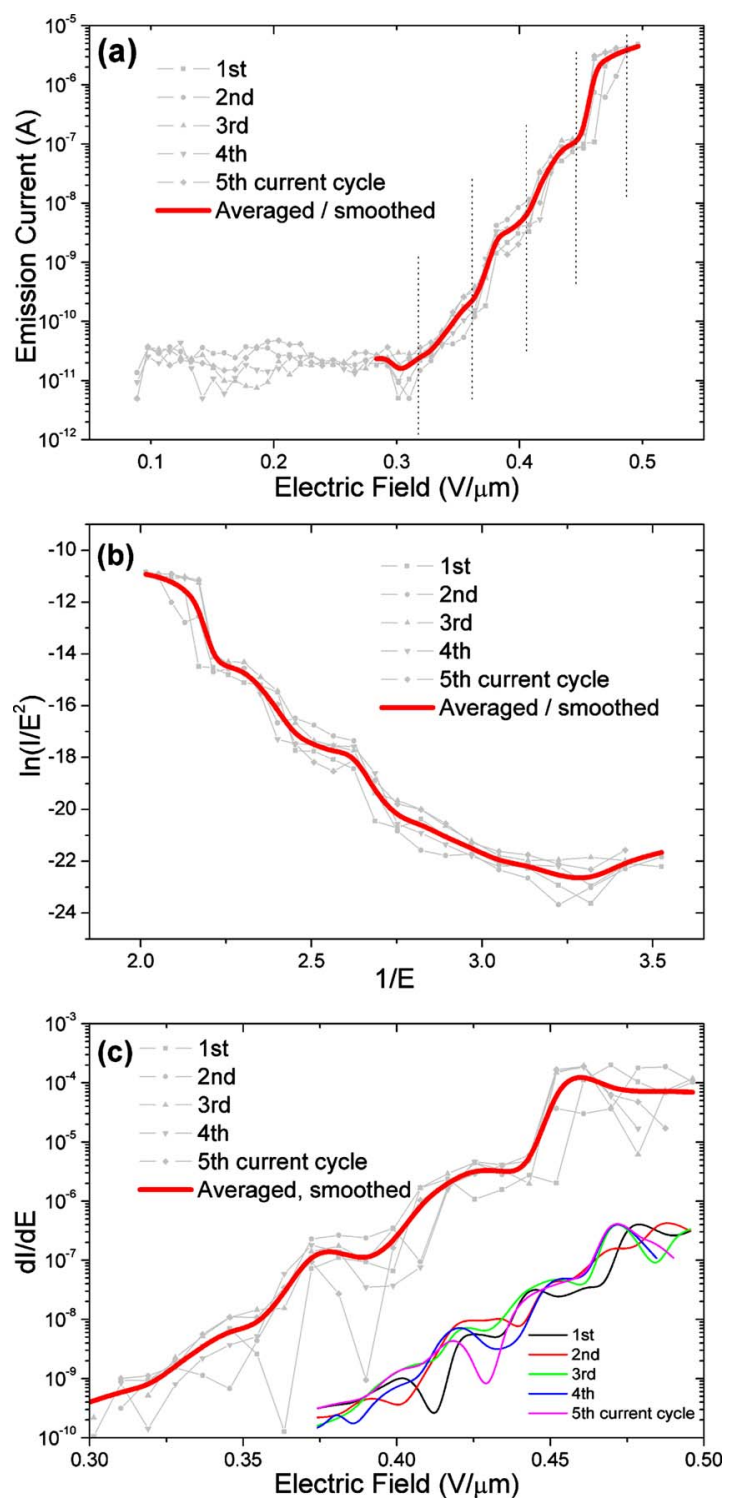

FIG. 2. (Color online) (a) Current-field curves for o-MWNTs on carbon fiber substrates (upward cycle only) and (b) the same data plotted in FN coordinates. (c) Derivative plot of the current-field data, highlighting the staircaselike nature of the data. The inset shows each cycle smoothed and shown separately for clarity.

originate from the o-MWNTs and not from the underlying carbon fiber.

Figure 2(a) shows FE current-field characteristics from the upward part of five current cycles, and Fig. 2(b) shows this data plotted in Fowler-Nordheim (FN) coordinates. The red line in each shows the averaged, smoothed data. The standard deviation in emission current is around $25 \%$, averaged over the whole emitting range. The current curves and the FN curves show staircaselike current-field characteristics, which are repeatable over the five successive voltage cycles. The current rises in steps of around one order of magnitude with a roughly constant step-width of $\sim 0.05 \mathrm{~V} / \mu \mathrm{m}$. These steps are highlighted in the derivative plot [Fig. 2(c)]. Figure 3 shows (a) the FE and (b) the FN characteristics for the downward part of the five cycles. These curves also have an atypical nonlinear shape. However, as opposed to the four regular steps of the upward part, these downward cycles have just two larger steps of unequal width.

A probable source of this nonlinearity is RT through quantized subbands at the MWNT surface. Nonlinearity can
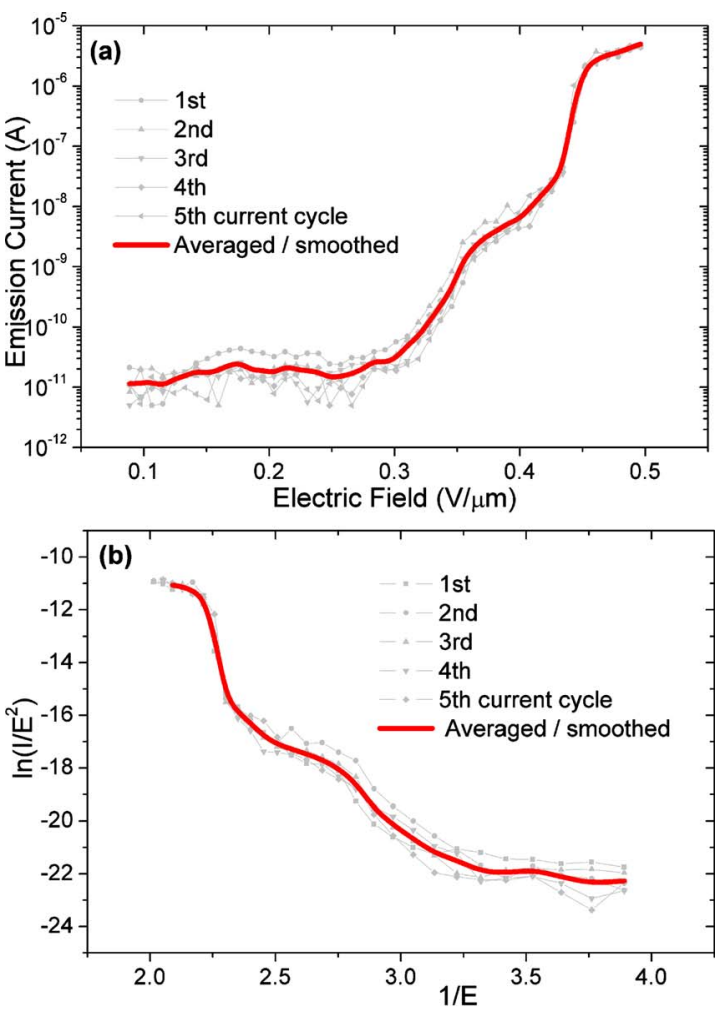

(c)

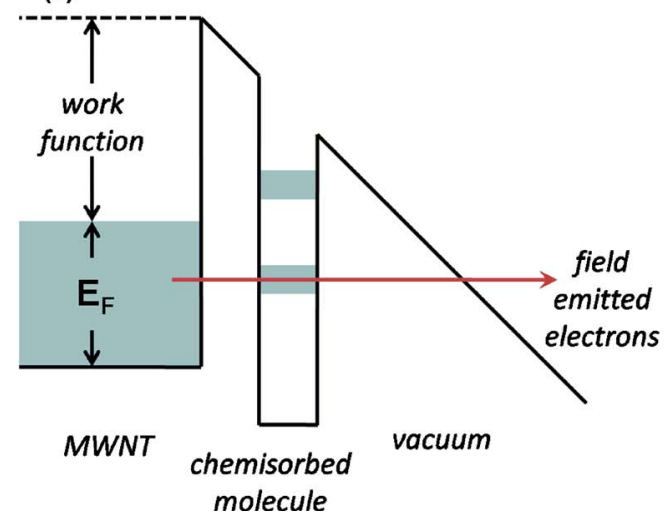

FIG. 3. (Color online) (a) Current-field curves for o-MWNTs on carbon fiber substrates (downward cycle only) and (b) the same data plotted in FN coordinates. (c) Idealized representation of resonance tunneling FE from a metal with a narrow band adsorbate.

arise in the FE current due to resonance when the electronic sublevels in quantum wells are aligned and negative differential resistance when misaligned. Theoretical FN plots of FE from $\mathrm{Si}-\mathrm{SiO}_{2}-\mathrm{Si}-\mathrm{SiO}_{2}$ cathodes have been attributed to RT (Ref. 3) and are strikingly similar to our experimental data, albeit with resonances at different field strengths. It is postulated that the nonlinearity in our experimental data is a result of a similar RT effect, with differences in the quantum well dimensions accounting for the disparity in resonance magnitudes. For RT to occur, quantum well structures must exist at the o-MWNT surface, the justification of which is outlined below.

It is suggested that the o-MWNT carboxylic moiety gives rise to a quantum well structure, providing the localized surface states necessary for RT to occur. Adsorbed water molecules may also contribute to this effect. For many years FE has been known to be strongly influenced by the existence of adsorbates; RT through the energy levels of atoms and molecules adsorbed on metal surfaces plays a major role 
in $\mathrm{FE}^{27}$ The existence of the attractive potential well of an atom close to the surface of a metal can change the shape and effective thickness of the tunneling barrier via resonant energy levels, dramatically increasing the tunneling probability. Figure 3(c) is an idealized potential diagram in which the potential of an adsorbate is taken as a square well. In this case, electrons are more likely to tunnel from metal to vacuum through the energy levels of the adsorbate due to a reduction in the effective thickness of the triangular barrier. Duke and Alferieff ${ }^{27}$ illustrated this by performing a onedimensional exact wave matching integral to evaluate FE transmission for a triangular barrier with various combinations of square wells and delta functions representing the adsorbate. They showed that adsorbates lead to wide resonances in the emission probability, an additional peak in the electron energy distribution at the resonant energy, large enhancement in the FE current, and reductions in the FN slope. The first experimental observation of RT through adsorbates was made by Plummer et al. ${ }^{28}$ in 1969 , as peaks in the energy distribution of electrons emitted from zirconium adsorbed on tungsten. This work led to the development of atomic spectroscopy for adsorbed atoms. Furthermore, in FE microscopy the emitted current will increase if an atom or molecule is adsorbed onto the local probe area due to RT enhancement. ${ }^{29}$ More recently, RT through localized surface states in chemisorbed molecules has been used to explain the lobed patterns observed in FE microscopy from CNT caps. ${ }^{29}$ As the temperature of the emitter increases, the lobes disappear and the emission current is reduced due to thermal removal of the adsorbates. RT through surface states is also widely observed and utilized in scanning tunneling microscopy. ${ }^{30-32}$

These RT effects have traditionally been observed via fine structure in the electron energy distribution of emitted electrons, whereby energy peaks visible in the energy spectra represent resonances. Otherwise, lobed patterns observed in FE microscopy have been explained by RT and scattering of electrons through adsorbates. ${ }^{33}$ However, fine structure in the FE current caused by localized surface states has rarely been reported, possibly due to the high field resolution necessary to resolve the individual resonances.

Given the theoretical predictions of RT in FE and the experimental observations of RT effects from surface adsorbates, it is likely that the experimental results presented here do indeed represent RT through the localized electronic energy levels of a chemisorbed molecule (i.e., the carboxyl moiety and/or adsorbed water molecules). The steps observed in the current-field data represent multiple electronic energy levels confined in the carboxylic potential well. As each energy level aligns with the Fermi level of the MWNT, an increase in the FE current is expected, as observed experimentally. The disparity between the up and down cycles can be explained by slow decharging due to trapped states in the quantum wells. These staircaselike characteristics have not been previously observed in the literature due to the very high field resolution necessary to resolve individual current steps (each being around $0.05 \mathrm{~V} / \mu \mathrm{m}$ wide). This was only achievable in these samples due to very low threshold fields. This work provides an insight into the underlying mechanisms of FE and as such could lead to applications in FE devices, for example, by tailoring chemisorbed and adsorbed atoms or molecules on surfaces to enhance the tunneling efficiency of electrons into vacuum, thereby improving FE efficiency.

In summary, staircaselike current-field characteristics were observed in FE from o-MWNTs. RT was explored as an explanation for this behavior, and published theory compared well with experiment. The carboxyl surface moiety is assumed to form a quantum well structure with multiple resonant energy levels. RT through these electronic subbands leads to quantized increases in the FE current with increasing field strength.

The authors would like to thank Lucian Dragos Filip and Wei-Mong Tsang for useful discussions and EPSRC (U.K.) for funding the research via the CBE and Portfolio Partnership awards.

${ }^{1}$ O. E. Raichev, Phys. Rev. B 73, 195328 (2006).

${ }^{2}$ T. J. Thornton, Low Dimensional Semiconductor Structures (Cambridge University Press, Cambridge, 2000), p. 327.

${ }^{3}$ Y. V. Kryuchenko and V. G. Litovchenko, J. Vac. Sci. Technol. B 14, 1934 (1996).

${ }^{4}$ V. G. Litovchenko, A. A. Evtukh, Y. M. Litvin, N. M. Goncharuk, and V. E. Chayka, J. Vac. Sci. Technol. B 17, 655 (1999).

${ }^{5}$ W. M. Tsang, S. J. Henley, V. Stolojan, and S. R. P. Silva, Appl. Phys. Lett. 89, 193103 (2006).

${ }^{6}$ L. V. Radushkevich and V. M. Luk'yanovich, Zh. Fiz. Khim. 26, 88 (1952).

${ }^{7}$ S. Iijima, Nature (London) 354, 56 (1991).

${ }^{8}$ M. M. J. Treacy, T. W. Ebbesen, and J. M. Gibson, Nature (London) 381 678 (1996).

${ }^{9}$ Z. W. Pan, S. S. Xie, B. H. Chang, C. Y. Wang, L. Lu, W. Liu, M. Y. Zhou, and W. Z. Li, Nature (London) 394, 631 (1998).

${ }^{10}$ Y. L. Li, I. A. Kinloch, and A. H. Windle, Science 304, 276 (2004).

${ }^{11}$ H. J. Dai, E. W. Wong, and C. M. Lieber, Science 272, 523 (1996).

${ }^{12}$ S. Berber, Y. K. Kwon, and D. Tomanek, Science 84, 4613 (2000).

${ }^{13}$ P. C. P. Watts, S. M. Lyth, E. Mendoza, and S. R. P. Silva, Appl. Phys. Lett. 89, 103113 (2006).

${ }^{14}$ P. C. P. Watts, N. Mureau, Z. Tang, Y. Miyajima, J. D. Carey, and S. R. P. Silva, Nanotechnology 18, 175701 (2007).

${ }^{15}$ W. Z. Li, S. S. Xie, L. X. Qian, B. H. Chang, B. S. Zou, W. Y. Zhou, R. A. Zhao, and G. Wang, Science 274, 1701 (1996).

${ }^{16}$ B. O. Boskovic, V. Stolojan, R. U. A. Khan, S. Haq, and S. R. P. Silva, Nature Mater. 1, 165 (2002).

${ }^{17}$ Q. H. Wang, A. A. Setlur, J. M. Lauerhaas, J. Y. Dai, E. W. Seelig, and R. P. H. Chang, Appl. Phys. Lett. 72, 2912 (1998).

${ }^{18}$ R. C. Smith and S. R. P. Silva, J. Soc. Inf. Disp. 16, 615 (2008).

${ }^{19}$ S. Uemura, SID Int. Symp. Digest Tech. Papers 32, 142 (2001).

${ }^{20}$ C. G. Lee, J. E. Jung, Y. W. Jin, S. H. Jo, S. J. Lee, J. H. Choi, N. S. Park, et al., SID Int. Symp. Digest Tech. Papers 33, 1125 (2002).

${ }^{21}$ M. S. P. Shaffer, X. Fan, and A. H. Windle, Carbon 36, 1603 (1998).

${ }^{22}$ E. Dujardin, T. W. Ebbesen, A. Krishnan, and M. M. J. Treacy, Adv. Mater. (Weinheim, Ger.) 10, 611 (1998).

${ }^{23}$ S. M. Lyth and S. R. P. Silva, Appl. Phys. Lett. 90, 173124 (2007).

${ }^{24}$ N. P. Blanchard, R. A. Hatton, and S. R. P. Silva, Chem. Phys. Lett. 434, 92 (2007).

${ }^{25}$ S. M. Lyth, R. A. Hatton, and S. R. P. Silva, Appl. Phys. Lett. 90, 013120 (2007).

${ }^{26}$ H. Ago, T. Kugler, F. Cacialli, W. R. Salaneck, M. S. P. Shaffer, A. H. Windle, and R. H. Friend, J. Phys. Chem. B 103, 8116 (1999).

${ }^{27}$ C. B. Duke and M. E. Alfereiff, J. Chem. Phys. 46, 923 (1967).

${ }^{28}$ E. W. Plummer, J. W. Gadzuk, and R. D. Young, Solid State Commun. 7, 487 (1969).

${ }^{29}$ K. A. Dean and B. R. Chalamala, J. Appl. Phys. 85, 3832 (1999).

${ }^{30}$ C. J. Chen, Phys. Rev. Lett. 65, 448 (1990).

${ }^{31}$ I. Martin, A. V. Balatsky, and J. Zaanen, Phys. Rev. Lett. 88, 097003 (2002).

${ }^{32}$ K. Bobrov, A. J. Mayne, and G. Dujardin, Nature (London) 413, 616 (2001).

${ }^{33}$ J. W. Gadzuk and E. W. Plummer, Rev. Mod. Phys. 45, 487 (1973). 